\title{
Image Recognition Algorithm and Sharpness Evaluation Based on Edge Width Detection
}

\author{
Yu Yuan ${ }^{1, a}$, YansongDeng ${ }^{1, b, *}$ \\ ${ }^{1}$ Institute of Electrical and Information Engineering, southwest Minzu University,West Terminal Street, \\ Chengdu,China \\ ${ }^{2}$ Department of communication Engineering, southwest Minzu University,West Terminal Street, \\ Chengdu,China

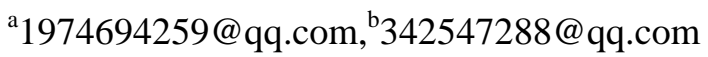 \\ *corresponding author
}

Keywords: Edge extraction; Local gradient extreme value; Double threshold selection; Image sharpness evaluation

\begin{abstract}
The edge is one of most significant features of the images, which is the basis of image analysis and recognition. When it comes to the segmentation, measurement of the object, the edge extraction and the noise resistance are of particular importance. In order to achieve the effective extraction of the image edge, this paper presents a new algorithm for the detection of image edge width. This method is based on the principle that the blurred image will lead to edge diffusion and is divided into two processing stages. The first step is aimed at calculating the maximum value of local gradients of the image, obtaining the rough edge information map of the image, and then obtaining the finer edge information map via the adaptive dual threshold selection method. In the second stage, the probability curve on the edge width of the graph is plotted, the skewness and variance are calculated, and then we can obtain the decline factor of the curve. Finally, we define the decline factor as the adjustment coefficient which is multiplied by the average width of the image edge and obtain the articulation value. Having analyzed the of sharpness of the image edge width which is extracted in both objective and subjective ways, the experimental results show that the edge width information extracted by this algorithm is close to the subjective evaluation of human eyes, further illustrating the feasibility of the proposed algorithm in this paper.
\end{abstract}

\section{Introduction}

In recent years, with the rapid development of digital information, images are used in daily life and work frequently, which may cause image quality degradation distortion in every step of image acquisition, transmission, storage and processing. The sharpness of the image edge is an important basis for measuring the quality of the image. Therefore, how to get the image edge information is very important.

The essence of edge detection is to use some algorithms to extract the boundary between the target and the background in the image. Traditional edge detection operators such as Sobel, Prewitt, Roberts and other operators are mostly local window gradient operators. However, these detection operators are not only very sensitive to noise, poor anti-noise ability, but also easily affected by noise. In 1986, John Canny proposed an optimal edge detection operator --- Canny operator. However, the Canny operator is too sensitive to noise, it is very easy to detect false edges, on the other hand, the contour edge of the target object is discontinuous. Thus, the processing result cannot be directly applied to the subsequent work such as segmentation and measurement. In order to come true it, this paper adopts a new edge-width detection algorithm, and a method of sharpness evaluation is proposed based on the image edge information on this algorithm. 


\section{Image edge contour extraction}

In this paper, we use the image edge detection method based on the Local gradient Maximum to extract the image edge. Due to there are a lot of points with large amplitude gradient in the image, which exist as edge noise in specific application fields. We need to determine which points are edge points, and the most concise edge detection criterion is the local gradient extreme value.

\subsection{Local gradient maximum}

The image edge is usually a collection of pixel points with step transitions or roof changes of pixel gray value around the image, mainly between the target and the background, the target and the target. In this paper, let us first assume that $I_{i, j}$ is the size of the grayscale at point $(i, j)$ in the $M N$ image. The grayscale of the image is $L$, and the grayscale value of point $I_{i, j}$ is in the range of $^{[0, L-1]}$. In order to obtain the edge detection image better, we select the center of the matrix with $I_{i, j}$ as $I_{i, j}$ and compare it with the surrounding 8-point gradient to get the local maximum value, so as to determine the image edge.

\begin{tabular}{|l|l|l|}
\hline 255 & 255 & 255 \\
\hline 255 & 255 & 0 \\
\hline 0 & 0 & 0 \\
\hline
\end{tabular}

Figure 1 Field grayscale

Gradient amplitude $\Psi\left(I_{i, j}\right)$ for each pixel in the image:

$$
\begin{aligned}
& \Psi\left(I_{i, j}\right)=\left\{\max \left|I_{i, j}-I_{x, y}\right|\right\} \\
& \quad(x=i-1, i, i+1 ; y=j-1, j, j+1)
\end{aligned}
$$

According to the above formula can get the maximum gradient within each neighborhood of the value, that is extract the rough edge of the image, as shown in Figure 2:

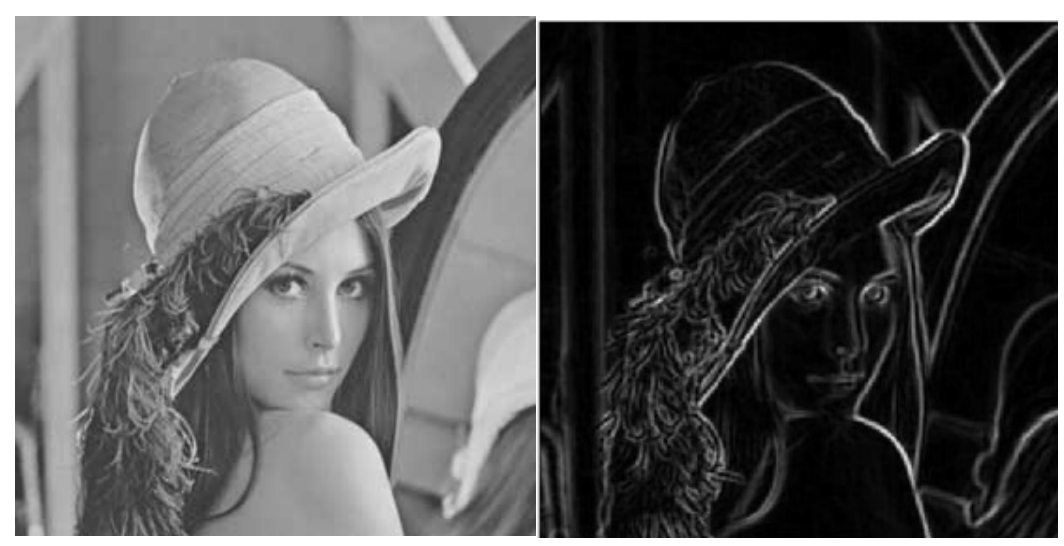

Figure 2 Image Edge Based on Local Gradient

\subsection{Adaptive threshold selection}

As can be seen from the rough edge in Figure 2, the edge pixels and non-edge pixels co-exist in the figure, and the isolated noise points will inevitably interfere with our evaluation of the edge width of the image. To filter out isolated noise, that is, non-edge pixels, let the edge set be separated from the non-edge set to obtain a clearer image edge width information map. Therefore, this paper chooses adaptive dual threshold selection method to filter out the non-edge set of images. 
In this paper, by selecting the best threshold, and using the threshold separation between the two classes so that it has better separation. The advantages and disadvantages of the separation between classes are judged by the largest difference in the statistical sense or the smallest difference in the characteristics of the class. Because there are also differences in characteristics between the two classes of true edge and non-edge sets, we combine the gradient histogram (Figure 3) with the classical Otsu algorithm, and choose the gradient variance to measure the two sets Rather than the originalOtsu algorithm will use the gradient mean as a measure. The improvedOtsu algorithm can adaptively determine the high threshold, and then the low threshold is determined by the high threshold, so that the adaptive selection of dual threshold is realized, and the two classes of edges are separated successfully.

It can be edge and non-edge set two categories of separation between the weights:

$$
\begin{aligned}
& \eta_{0}(t)=W_{0}(t)\left(\sigma_{0}^{2}(t)-\sigma^{2}\right)^{2} \\
& \eta_{1}(t)=W_{1}(t)\left(\sigma_{1}^{2}(t)-\sigma^{2}\right)^{2}
\end{aligned}
$$

Adaptive Threshold Selection:

$$
T=\operatorname{ArgMax}\left[\eta_{0}(t)+\eta_{1}(t)\right]
$$

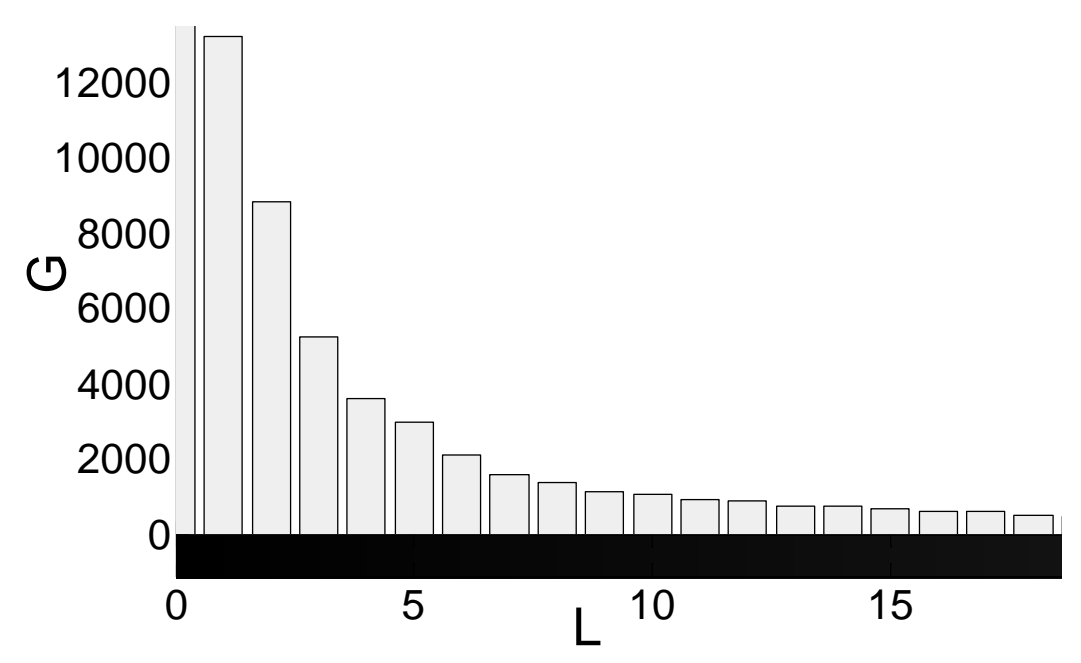

Figure 3 Gradient chart

Among them:

$$
\begin{gathered}
W_{0}(t)=\sum_{0<i \leq t} p(i) \\
W_{1}(t)=\sum_{t<i \leq L-1} p(i) \\
u_{0}(t)=\sum_{0<i \leq t} \frac{i p(i)}{W_{0}(t)} \\
u_{1}(t)=\sum_{t<i \leq L-1} \frac{i p(i)}{W_{1}(t)} \\
\sigma_{0}^{2}(t)=\frac{1}{W_{0}(t)} \sum_{0 \leq i \leq t}\left(u-u_{0}(t)\right)^{2} p(i)
\end{gathered}
$$




$$
\sigma_{1}^{2}(t)=\frac{1}{W_{1}(t)} \sum_{t<i \leq L-1}\left(u-u_{1}(t)\right)^{2} p(i)
$$

Specific methods for determining double threshold value:

- According to the gradient histogram, if the high threshold is set, the rough edge image can be divided into an edge point set and a non-edge point set;

- Calculate the probability of aggregated pixel occurrences $w_{0}(t)$ and $w_{1}(t)$, as well as the mean of all gradient values, $u_{0}(t)$ and $u_{1}(t)$, of each gradient.

- Calculate the gradient variance of edge pixels and non-edge pixels separately, $\sigma_{0}^{2}$ and $\sigma_{1}^{2}$, and all pixels have a gradient of $\sigma^{2}$.

- According to the above formula to determine the $T_{H}$, low threshold we use the method of estimating the classical Canny operator, that $T_{L}=0.4 T_{H}$.

- Determine the point where the gradient value is greater than the threshold as the edge point in the image rough edge information map, discard the point whose gradient value is less than the low threshold value, and if the pixel point between the two thresholds is in communication with other edge points, Point, otherwise discarded. Select the results shown in Figure 4.

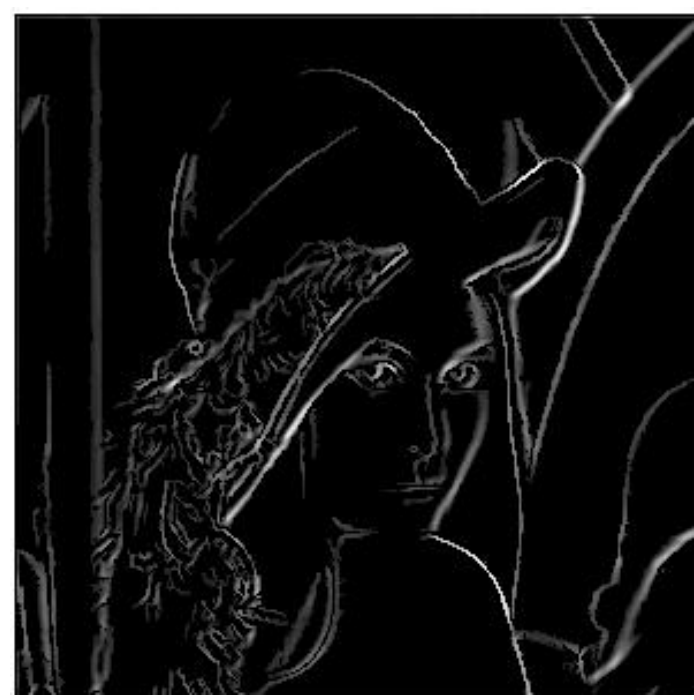

Figure 4 dual threshold processing
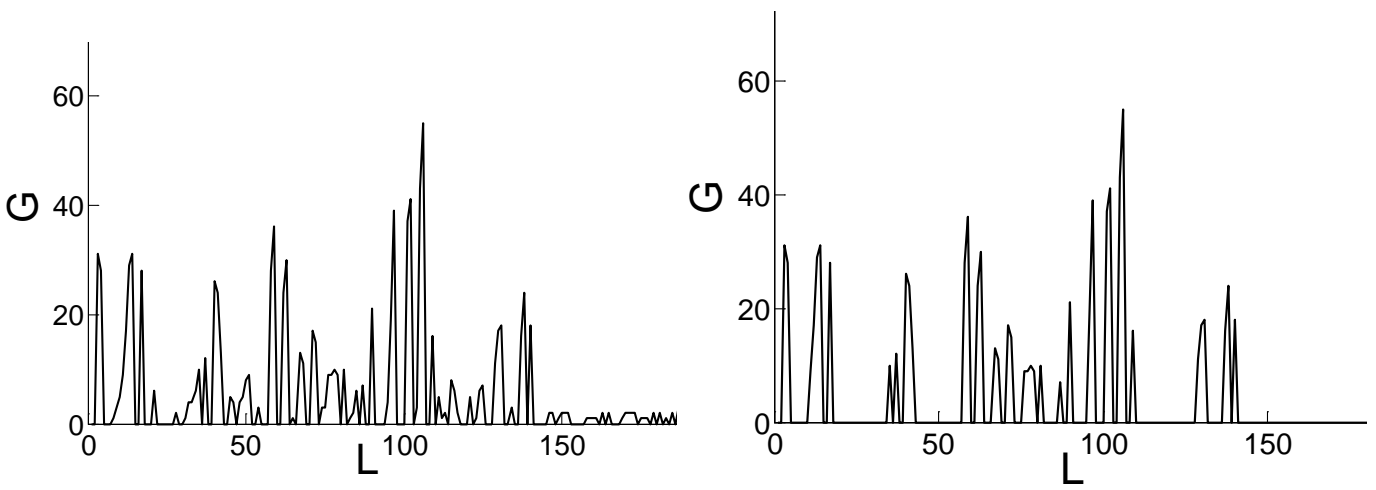

Figure 5 Double thresholding before and after the image line

After the double thresholding image is selected (Figure 4), the isolated noise points are effectively screened out compared to the rough edge information (Figure 2). By comparing the gray values of the same line before and after the double threshold of the edge image in Figure 5, it can be seen that the adaptive double threshold selection effectively filters out the vast majority of isolated noise points and non-edge points in the edge image, The integrity of the edge of the image, which will help improve the accuracy of image sharpness evaluation. 


\section{Image sharpness evaluation}

After the adaptive double threshold selection, the edge of the image with clear image feature points is obtained. After analysis the step-by-step character of the grayscale profile in the edge region of the image, a dramatic change from a grayscale value in a small buffer region to a high grayscale region. Therefore, when calculating the width of the edge, first search the extreme value of a certain line in the image, and according to the definition of the edge, find the maximum between the two adjacent minimum values, as shown in the following figure The minimum value of PA1 and PA3 respectively, the maximum value of PA2, the width of the edge is D = PA3-PA1.

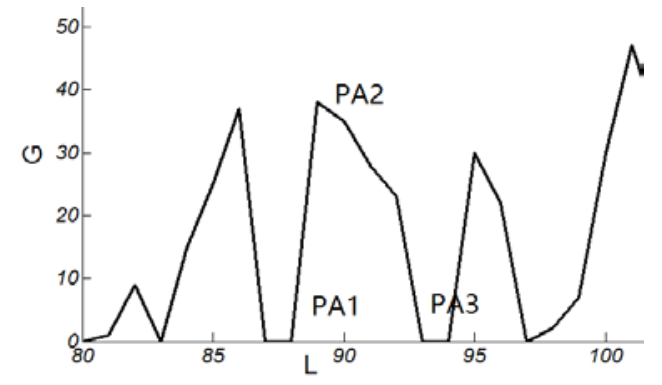

Figure 6 One line of the edge image

Figure 7 makes a different degree of ambiguity for the three pictures, and then searches all the edge points in the horizontal direction, and calculates the probability of all the widths

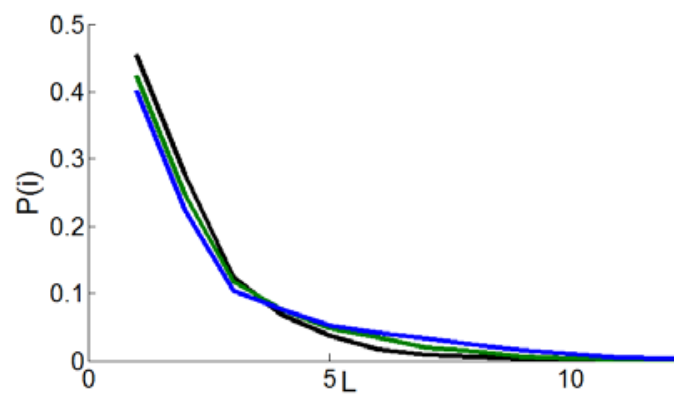

Figure 7 Width $L$ - probability $p(i)$ curve

The width-probability curve distribution of the above image can observe the degree of curve skew, the degree of tailing and the variance reflecting the variance of the average digital features. The curve of a large number of images after Gaussian blurring is statistically analyzed and analyzed. As the blurring effect aggravates, the width-probability curve tends to be flat and the peak part descends and shifts to the right.

Obviously, these changes are due to blurring of the step or near-step edges. To more accurately reflect the ambiguity, we set and introduce a curve descent factor based on the obvious local features and overall information of the graph:

$$
S / \sigma
$$

Where $\sigma$ is the variance of the image, and $S$ is the standard third-order moment for the distribution, which is the skewness of the width probability curve of the entire image. Skewness is a statistic that describes the distribution symmetry of a variable's value, and $\mu$ is the mean of the curve's probability.

$$
S(p(i))=E\left[\left(\frac{p(i)-\mu}{\sigma}\right)^{3}\right]
$$

The curve descent factor can directly explain the descending speed of the width probability curve, that is, the bigger the factor, the faster the curve descends. This factor can also effectively reflect the contribution of the width information of the entire image to the definition index, instead of being 
limited to only one of the features, enhancing the overall image data characteristics and reducing the edge width corresponding to the ineffective edge or noise to the sharpness index Interference.

Referring to the principle of entropy of information in Shannon's Information Theory, the curve factor (Eq. 11) is introduced into the weighted average edge width model to get the definition evaluation index of this paper:

$$
M t=\log \left[\sum \frac{S}{\sigma} \cdot i \cdot P(i)\right]
$$

The evaluation index shows that the more blurred the image, the larger the evaluation value. Select lena original map in Figure 1, the Gaussian blur with a variance of 0.1 steps, followed by 0 to 3 , calculate the resolution of thirty different degrees of ambiguity image evaluation index. The following figure clearly observed the image evaluation value and the degree of fuzzy linear relationship shows that the evaluation of this article is more reasonable.

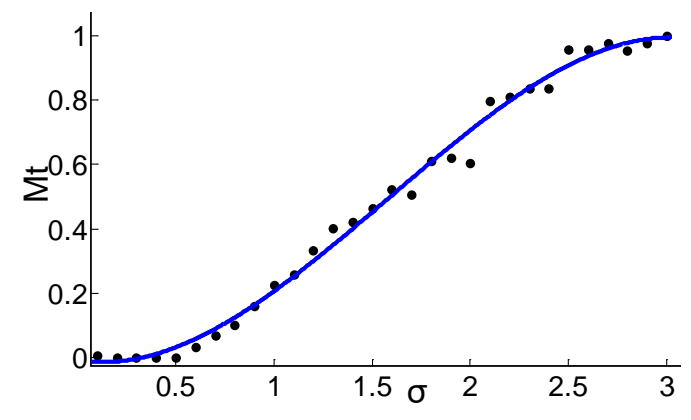

Figure 8 Clarity Mt and degree $\sigma$ curve

\section{Experimental analysis}

\subsection{Subjective and objective consistency of the experiment}

In this paper, LIVE database Gaussian blur image library selection of the picture sharpness evaluation index $M t$ and subjective evaluation of the average fitting, the fitting equation can be obtained:

$$
\mathrm{V}_{\mathrm{i}}=\frac{\alpha_{1}}{\alpha_{2}+\exp \left(\alpha_{3} * M \mathrm{t}_{\mathrm{i}}^{\beta}\right)}+\alpha_{4}
$$

Where: $M t$ is the sharpness evaluation value, $V_{i}$ is the subjective evaluation value, $\alpha_{1} \sim \alpha_{4}$ and $\beta$ are the model parameters to be fitted.

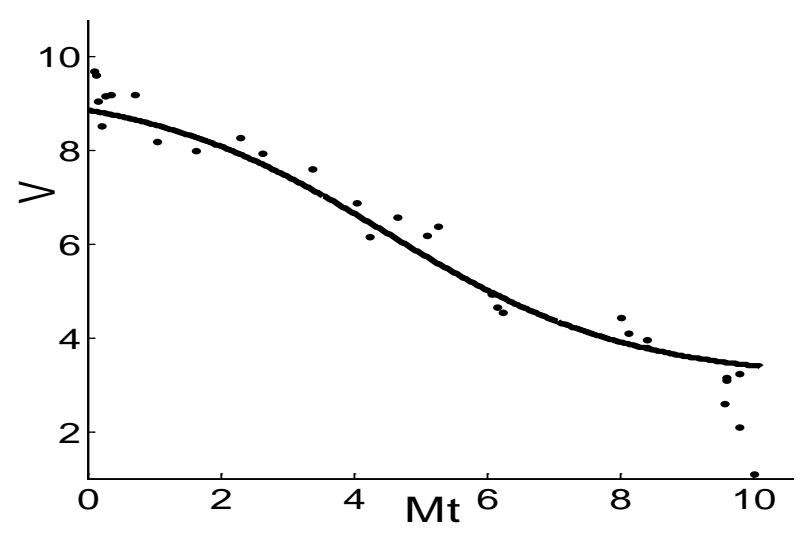

Figure 9 The subjective and objective consistency model curve 
Table 1 fitting parameters

\begin{tabular}{|c|c|c|c|c|}
\hline method & SSE & RMSE & $\begin{array}{c}\text { R- } \\
\text { square }\end{array}$ & Adjusted R-square \\
\hline article & 12.67 & 0.712 & 0.9333 & 0.9226 \\
\hline
\end{tabular}

In order to evaluate the practicability of the model, the parameters SSE and SSE based on the error between the fitting value and the subjective evaluation value are selected, and the error parameters $R$-square and Adjusted $R$-square of the subjective evaluation mean are selected.

The sum of squared errors (SSE) describes the square sum of the fitted error and the corresponding point error of the subjective evaluation. The smaller the square root of the mean ( $R M S E$ ) indicates that the closer the evaluation model is to the subjective evaluation. The deviation squared sum $(R$-square $)$ characterizes a good or bad by the change of data. The general value range is $\left[\begin{array}{ll}0 & 1\end{array}\right]$, the closer it is to 1 , indicating that the objective model of the equation is more capable of interpreting the curve. The model described in the above table is very close to the subjective evaluation.

\subsection{Motion blur experiment}

The above experiments all test the performance of the algorithm under Gaussian blur, that is, out of focus. According to the principle of the algorithm detecting the edge contour, the method is also applicable to the situation of motion blur. In the underwater robot pipeline inspection project, the algorithm is used to detect the number of oil spill points in the pipeline. Select picture 9 as the test object. Motion blur in 1 pixel steps, followed by 1 to 20 pixels, blur direction for the $45^{\circ}$ direction, the test results shown in Figure 10, can bearish As the amount of motion blur increases, the evaluation value becomes larger, consistent with the expectations.

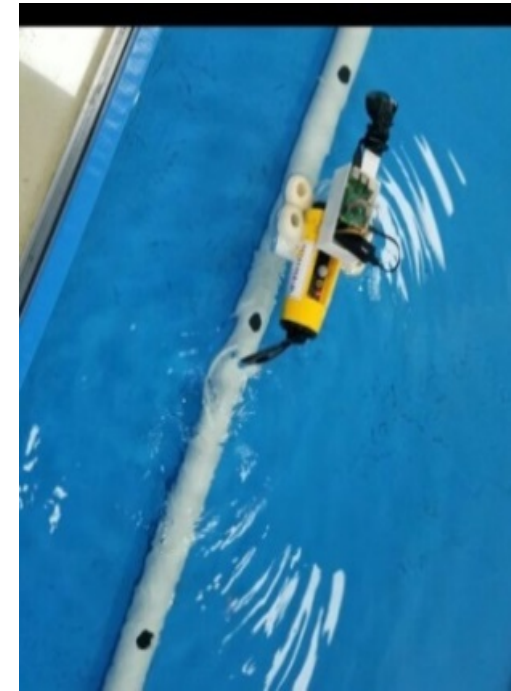

Figure 10 Motion blur experiment to

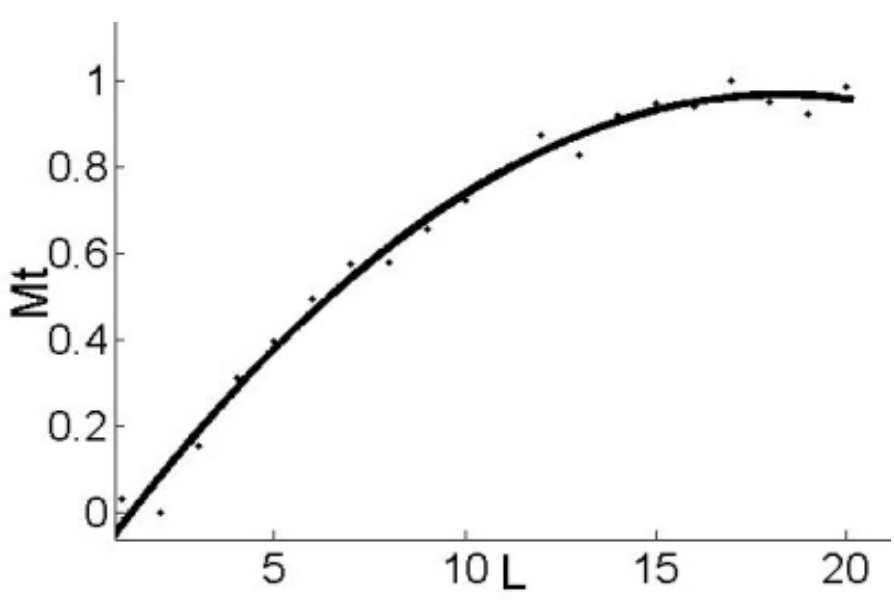

Figure 11 Test Results Analysis

Evaluate the image 


\section{Experimental comparison}

In order to verify the feasibility of the edge width detection algorithm in this paper, the algorithm is implemented on the Matlab platform, In the traditional Canny edge detection algorithm, the standard deviation $\alpha$ of the Gaussian filter is set to 1 , and the high and low thresholds are respectively 150 and 70 . Select the following two respectively, the comparison results are as follows:

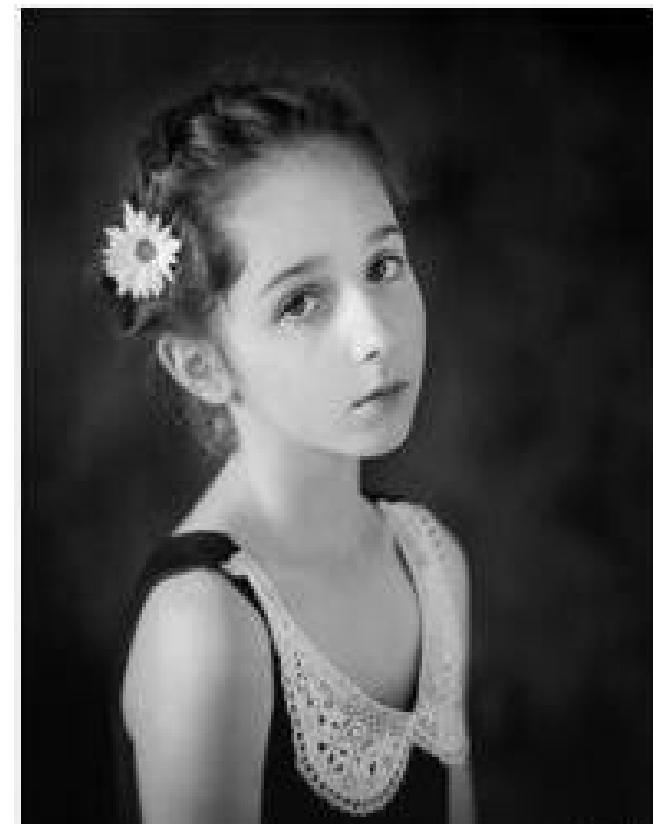

Figure a

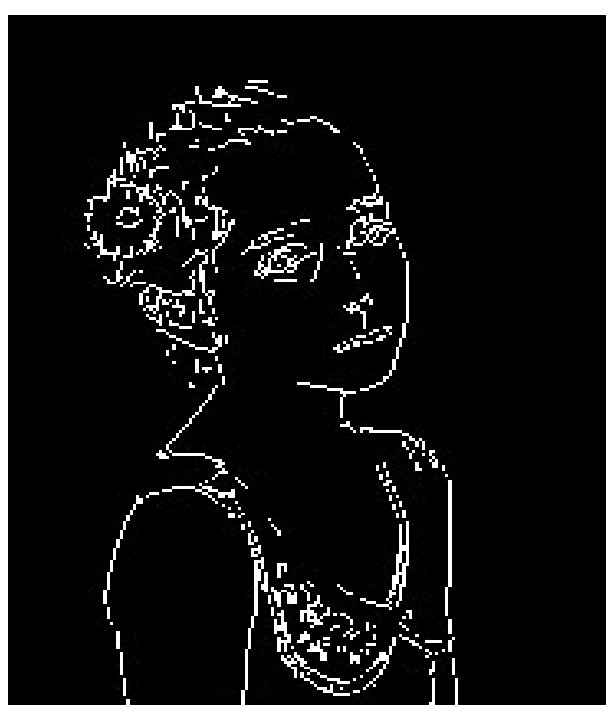

Figure c

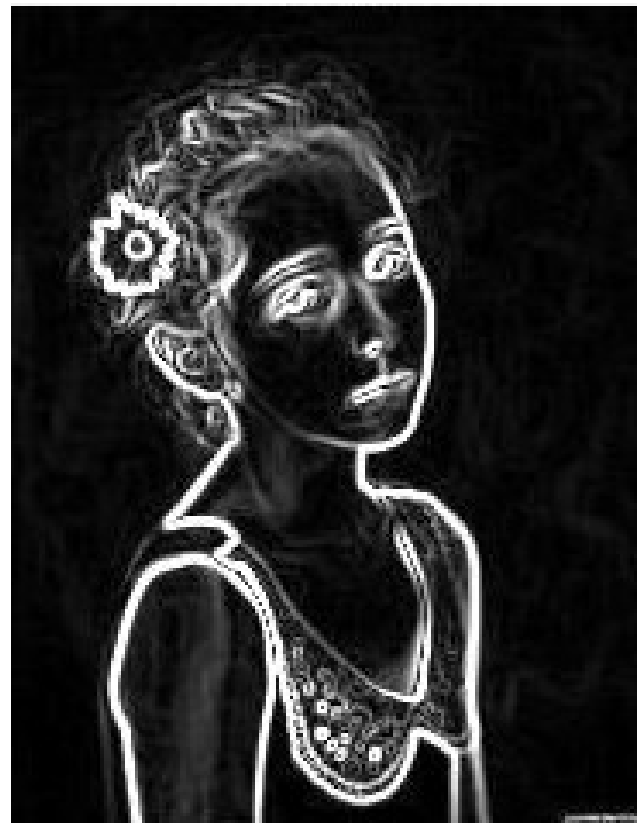

Figure b

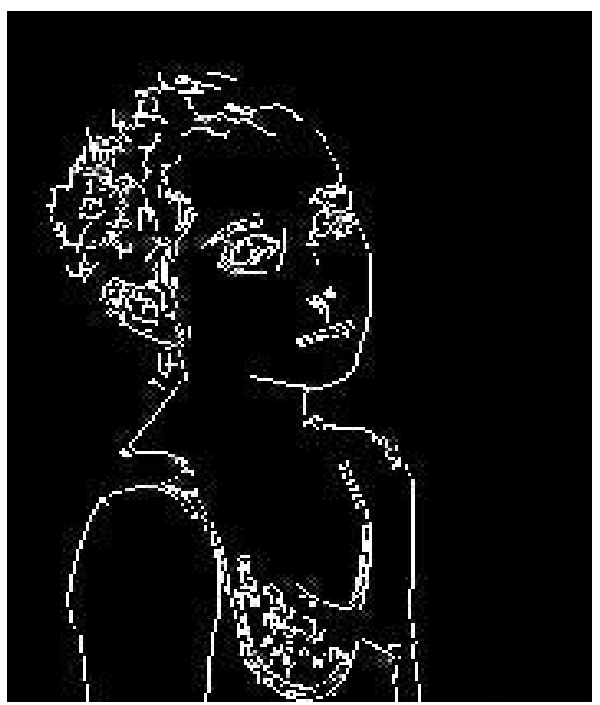

Figure d

Figure a is the original image, Figure b is the edge information obtained by local maximum, Figure $\mathrm{c}$ is the final edge information image obtained by adaptive double threshold filtering, Figure $\mathrm{d}$ is a picture obtained by the traditional Canny algorithm. It is easy to see that the edge image extracted by the traditional Canny algorithm has been lost and cannot be guaranteed. Go one step further, the edge image extracted by the algorithm in this paper which is more detailed and accurate than the edge image obtained by the traditional Canny algorithm. In short, it can be seen that the proposed algorithm in this paper has good accuracy, completeness and sharpness. 


\section{Conclusion}

Image edge extraction has always been the vital subject of image processing field. This paper introduces the concept of local maximum, and through analysis of it, a new method is proposed on the basis of local gradient extreme value and adaptive threshold selection, which can extract the edge of the image. Finally, by analyzing the sharpness, we discover that the evaluation index and image content are almost independent, and the evaluation results are in line with the subjective evaluation of human eyes, its low computational complexity is suitable for practical application. However, what is necessary to point out is that this algorithm cannot be applied to the image containing strong salt and pepper noise. It is a further meaningful task to propose a more efficient edge extraction algorithm, which can extract the edge information of the step edge or the edge information of the class edge effectively.

\section{References}

[1] Zeng Jun, Li Dehua et al., Image Edge Detection Technology and Application Research, Control Science and Engineering, 2011.10.24

[2] R.C.Gonzalez, R.E.Woods. Digital image processing (second edition). Ruan Qiuqi, Ruan Yuzhi other translation, background: Point Electronic Industry Press .2003

[3] Gatti, MENG Xiangfu, MENG Lu, DONG Na, FANG Jinhe Image edge extraction based on Gaussian weighted distance graph, Journal of the Chinese Society of Graphic and Graphic Arts.2014,19 (1): 62-68

[4] Wang Zhi, He Saixian, Adaptive edge detection based on Canny theory [J] .Journal of Image and Graphics, 2004,9 (8): 957-961

[5] Hong Wensong, Chen Wufan.Generalized fuzzy operator method for image edge detection [J] .Journal of Image and Graphics, 1999,4 (2): 143-146

[6]Gonzalez RC,WoodsRE.Digital Image Processing(Third Edition) [M].Beijing Publishing House of Electronices Industry,2011:445-465.

[7] Wang Xiangju, based on median filtering and wavelet transform image denoising method, computer application technology, 2008.06.10

[8] Zhang Yuanlin, Zheng Nanning, Yuan Zejian, an improved image adaptive non-linear filtering method, Journal of Xi'an Jiaotong University, 38: 16-166,2004. 